\title{
Macroscopic Stability of High $\beta$ MAST plasmas
}

\author{
IT Chapman ${ }^{1}$, RJ Akers ${ }^{1}$, WA Cooper ${ }^{2}$, AR Field $^{1}$, JP \\ Graves $^{2}$, MP Gryaznevich ${ }^{1}$, RJ Hastie ${ }^{1}$, TC Hender ${ }^{1}$, DF \\ Howell $^{1}$, M-D Hua ${ }^{1,3}$, GTA Huysmans ${ }^{4}$, YQ Liu ${ }^{1}$, HF Meyer ${ }^{1}$, \\ CA Michael ${ }^{1}$, G Naylor ${ }^{1}$, SD Pinches ${ }^{1}$, S Saarelma ${ }^{1}, \mathbf{R}$ \\ Scannell $^{1}$, SA Sabbagh ${ }^{5}$, and the MAST Team \\ ${ }^{1}$ Euratom/CCFE Fusion Association, Culham Science Centre, Abingdon, OX14 \\ 3DB, UK \\ ${ }^{2}$ CRPP, Association EURATOM/Confédération Suisse, EPFL, 1015 Lausanne, \\ Switzerland \\ ${ }^{3}$ Imperial College, Prince Consort Road, London, SW7 2BY, UK \\ ${ }^{4}$ CEA-Cadarache, Association Euratom-CEA, 13108 St Paul-lez-Durance, France \\ ${ }^{5}$ Department of Applied Physics and Applied Mathematics, Columbia University, \\ New York, NY, USA \\ E-mail: ian.chapman@ccfe.ac.uk
}

\begin{abstract}
The high-beta capability of the spherical tokamak, coupled with a suite of world-leading diagnostics on MAST, has facilitated significant improvements in the understanding of performance-limiting core instabilities in high performance plasmas. For instance, the newly installed Motional Stark Effect (MSE) diagnostic, with radial resolution $<25 \mathrm{~mm}$, has enabled detailed study of saturated long-lived modes in hybrid scenarios. Similarly, the upgraded Thomson Scattering (TS) system, with radial resolution $<10 \mathrm{~mm}$ and the possibility of temporal resolution of $1 \mu \mathrm{s}$, has allowed detailed analysis of the density and temperature profiles during transient activity in the plasma, such as at a sawtooth crash. High resolution Charge Exchange Recombination Spectroscopy (CXRS) provided measurement of rotation braking induced by both applied magnetic fields and by magnetohydrodynamic (MHD) instabilities, allowing tests of neoclassical toroidal viscosity theory predictions. Finally, MAST is also equipped with internal and external coils that allow non-axisymmetric fields to be applied for active MHD spectroscopy of instabilities near the no-wall beta limit. Such resonant field amplification measurements suggest that MAST has been able to operate above the no-wall limit. In order to access such high pressures, the resistive wall mode must be damped, and so numerical modelling has focussed on assessing the kinetic damping of the mode and its nonlinear interaction with other instabilities. The enhanced understanding of the physical mechanisms driving deleterious MHD activity given by these leading-edge capabilities has provided guidance to optimise operating scenarios for improved plasma performance.
\end{abstract}




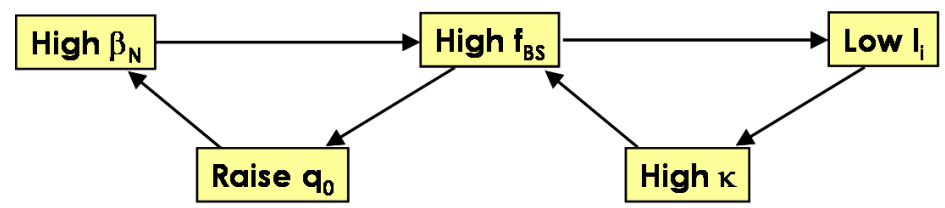

Figure 1. Virtuous cycle when high $\beta_{N}$ plasmas are accessed; giving rise to enhanced bootstrap fraction, allowing lower internal inductance and increased shaping which further enhances the non-inductively driven fraction, allowing the safety factor on axis to be raised, in turn permitting higher pressure plasmas.

\section{Introduction}

The ultimate goal of magnetic confinement fusion research is to produce steady-state burning plasmas in a fusion power plant. In order to achieve this, it is necessary to develop a plasma scenario which optimises the ratio of the plasma energy to the magnetic field energy (ie maximise $\beta$ where $\beta=2 \mu_{0}\langle p\rangle / B_{0}^{2}$ and $\langle\cdots\rangle$ represents an averaging over a flux surface, $p$ is the plasma pressure and $B_{0}$ is the toroidal magnetic field) whilst simultaneously minimising the amount of power required to supply the current non-inductively. Indeed, the EU Power Plant Conceptual Study (PPCS) [1] concluded that in order to produce electricity at economically attractive rates in a future fusion power plant, plasma performance beyond the ITER baseline level [2] is required [3]. Furthermore, high temperature, and consequently high $\beta_{N}$, plasmas are also desirable to enhance current drive efficiency and increase synchrotron radiation, reducing the power load on the divertor [4]. Previous power plant optimisation studies [5] concluded that the cost of electricity scales as

$$
\text { c.o.e. } \propto\left(\frac{D F}{A}\right)^{0.6} \frac{1}{\eta_{t h}^{0.5} P_{e}^{0.4} \beta_{N}^{0.4} N^{0.3}}
$$

where $D$ is the discount rate, $F$ is the tenth of a kind factor, $A$ is the plant availability, $\eta_{t h}$ is the thermodynamic efficiency, $P_{e}$ is the unit size, $N$ is the normalised density and $\beta_{N}$ is the normalised plasma beta, $\beta_{N}=\beta a B_{0} / I_{p}$ where $a$ is the minor radius and $I_{p}[\mathrm{MA}]$ is the plasma current. Equation 1 indicates that the cost of electricity will decrease as the normalised beta increases. Furthermore, enhancing the plasma beta leads to a virtuous feedback on plasma performance (illustrated in figure 1): High $\beta$ leads to a larger fraction of the plasma-generated non-inductive bootstrap current [6], which in turn lowers the internal inductance $\left(l_{i}\right)$ of the plasma. Operating at lower inductance permits stable operation at higher elongation $(\kappa)$. This in turn enhances the bootstrap fraction $\left(f_{B S}\right)$ further. The increased bootstrap fraction means that more current is driven off-axis, which, for a fixed total current, raises the safety factor at the magnetic axis, $q_{0}$. By increasing the safety factor above unity, typically the plasma stability is improved, allowing operation at higher $\beta_{N}$. Consequently, it is highly desirable to operate the plasma at the highest possible normalised beta to improve fusion performance.

'Steady state scenarios' [7-11] maximise the self-generated non-inductively driven bootstrap current [6] through a combination of operating at high plasma pressure and 
low plasma current. However, since the energy confinement degrades with decreasing plasma current, the energy confinement must be optimised in order to operate at economically-attractive fusion performance. Furthermore, high plasma pressures and low currents can lead to deleterious magnetohydrodynamic (MHD) instabilities which would not be unstable with conventional H-mode profiles [12]. Consequently, it is of considerable interest to understand the implications for MHD stability in regimes optimised for continuous operation.

The two main candidate scenarios for continuous tokamak operation are the Advanced Tokamak steady-state scenario [11], which has reversed magnetic shear in the core, low internal inductance and $\beta>\beta^{\text {nowall }}$, and the 'hybrid' stationary scenario $[13,14]$, which has a broad low shear region with the safety factor, $q$, above unity, a moderate internal inductance, $\beta \sim \beta^{\text {nowall }}$ and is expected to operate for long pulse duration in a quiescent state in ITER (where the magnetic shear is $s=r / q \mathrm{~d} q / \mathrm{d} r$ ). The reversed shear (or broad low shear in the case of the hybrid scenario) is a consequence of the optimised non-inductively driven currents, both from auxilliary current drive actuators and from the bootstrap current which is naturally driven off-axis in regions of strong pressure gradients. Combining such broad current profiles with the peaked pressure profiles required for enhanced plasma performance, can result in increased susceptibility to ideal $n=1$ kink-ballooning modes, or 'infernal modes' $[11,12,15]$. Indeed, even if the hard $\beta$-limit (ie the occurrence of a resistive wall mode (RWM)) is not encountered, other MHD instabilities, such as infernal modes or neo-classical tearing modes (NTMs) often inhibit plasma performance at high pressure [16]. Whilst these instabilities can be avoided by specific tailoring of the plasma profiles and careful navigation of trajectories in operational space, it is important to understand the domains of operation where such MHD instability will occur.

Recent MAST experiments have focussed on improving the understanding of these instabilities and their evolution; a process facilitated by sophisticated modelling of plasma stability. MAST plasmas with hybrid scenario q-profiles often exhibit longlived saturated ideal instabilities which cause significant rotation braking, degrade confinement and enhance fast ion losses. The stability of these long-lived modes (LLMs) is investigated in section 2, whilst the braking in the presence of the mode, as well as braking due to applied magnetic fields, is compared to predictions from neoclassical toroidal viscosity theory in section 3. High resolution Thomson scattering measurements have been used to examine the evolution of the magnetic island structure at a sawtooth crash - the antecedent of neoclassical tearing modes (NTMs) that degrade performance - as discussed in section 4. Finally, if the profiles are tailored in such a way as to avoid NTMs and LLMs, the ultimate $\beta$-limit is set by resistive wall modes. RWM stability has been probed by resonant field amplification and the role of kinetic damping in MAST is explored in section 5. By testing stability modelling against experimental data from high resolution diagnostics, credence is given to the stability testing of plasmas foreseen following an upgrade to MAST, as described in section 6. 

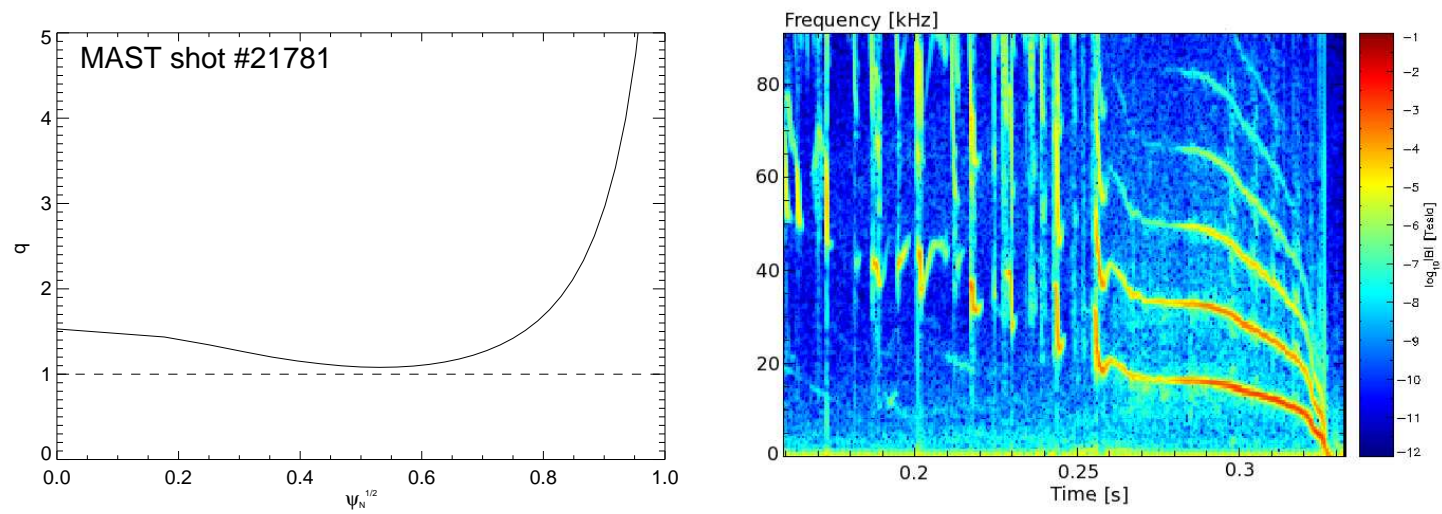

Figure 2. (a) The $q$-profile evolution in MAST shot 21781; (b) The Fourier spectrogram of outboard midplane magnetic measurements showing chirping modes which transition into a long-living saturated mode

\section{Saturated ideal modes in advanced tokamak regimes}

In order to investigate stability in plasmas with advanced tokamak scenario profiles, MAST typically operates with early NBI heating and low density. By applying early heating, the temperature increases and consequently the plasma resistivity decreases, meaning that the current diffusion time lengthens. As a result of this, the inductively driven current takes sufficiently long to diffuse into the core that the safety factor profile is naturally reversed, or has broad weak shear, with $q_{\text {min }} \sim 1$ for the majority of the discharge. Routine operation at relatively low density is compatible with achieving advanced tokamak $q$-profiles, whilst a monotonic $q$-profile with $q<1$ is obtained when the plasma density is increased. During discharges with such hybrid-like $q$-profiles, the motional stark effect (MSE) diagnostic is used to measure the pitch angle of the field lines and provide accurate $q$-profile reconstruction with good temporal $(<0.5 \mathrm{~ms})$ and spatial $(\sim 2.5 \mathrm{~cm})$ resolution $[17,18]$.

Hybrid plasmas in MAST with a safety factor above unity and a $q$-profile with either weakly reversed shear, or broad low shear regions (as illustrated in figure 2a) regularly exhibit long-lived saturated ideal MHD instabilities $[19,20]$. Frequency sweeping $n=1$ modes are observed to precede a saturated mode (figure $2 \mathrm{~b}$ ) whose initial frequency is close to that of the central rotation measured by charge exchange recombination spectroscopy. These ideal modes, distinguished as such by the notable lack of islands or signs of reconnection [20], are driven unstable as the safety factor approaches unity. In the presence of such ideal perturbations, the toroidal rotation profile is flattened and the fast ion losses are enhanced. Simulation of the fluctuations of SXR data induced by the mode show that the eigenstructure of the LLM is consistent with that of an $(m, n)=(1,1)$ internal kink mode.

Theory analysing the reversed shear $q$ profile indicates that it is prone to the $(m, n)=(1,1)$ internal kink mode [21]. The mode is unstable, even at zero $\beta$, for $\Delta q=q_{\text {min }}-1$ under a critical value $\Delta q_{\text {crit }}$ calculated analytically for low inverse aspect 
ratio plasmas in [21]. This mode saturates non-linearly if the $q$ profile remains above 1 [22]. Since the instability is ideal, the saturation occurs when the stabilising field line bending term balances the mode's fluid drive. Theory focusing on flatter core $q$ profiles predicts such plasmas to be unstable to low $(m, n)$ internal kink-ballooning instabilities, or infernal modes [15,23], with the most unstable modes having $m=n$ when $q_{\min } \sim 1$. These modes also feature a critical value of $\Delta q$ under which they are destabilised $[21,23]$. This critical value decreases with the $n$ number, whereas the mode's growth rate increases with it at $\Delta q \sim 0$.

The linear stability of the $n=1$ internal mode has been assessed for the evolving $q$-profile exhibited in MAST plasmas. The $q$-profile has been reconstructed at many timeslices for a number of discharges by constraining the EFIT equilibrium reconstruction with MSE field line pitch angle measurements and the magnetic probe measurements on the inboard side. The current profile and plasma shape are then supplied to the HeLENA equilibrium code [24] together with the pressure profile shape derived from the high resolution Thomson scattering measurements of the electron temperature and density. The linear stability of this equilibrium is then tested using the CAstor MHD code [25]. The ideal $n=1$ internal mode is found to be unstable for an equilibrium reconstructed at, or near, the same time as the LLM appears experimentally, but stable beforehand. Two example discharges are shown in figure 3. These plasmas have different toroidal field and heating schemes, meaning that $\Delta q$ reaches the critical threshold for the LLM to become unstable at different times. However, in both cases the numerical stability analysis predicts the infernal mode to become unstable for equilibria reconstructed at approximately the times of the observed LLM onset. Figure 4 shows the time at which the long-lived mode appears experimentally with respect to the time of equilibrium reconstruction at which the ideal internal $n=1$ mode is found to be unstable for a number of randomly-selected MAST discharges. This suggests that the mode onset can be well described by ideal MHD. Rotational and kinetic damping effects of these infernal modes are found to result in only a small change in the stability limits [19].

The transition from the chirping modes to the saturated mode can also be explained by the safety factor evolution. As the current diffuses into the core and the safety factor evolves downwards, MAST plasmas usually experience a phase of $n=1$ fishbone activity before the LLM appears, as illustrated in figure 2. The dispersion relation for fishbones with a non-monotonic $q$-profile and assuming a slowing down distribution, $F_{0 h}=c_{0} E^{-3 / 2} \delta\left(\lambda-\lambda_{0}\right)$ where $c_{0}(r)$ is defined in reference [26] was derived in reference [19] as

$$
\begin{aligned}
& \delta \hat{W}_{f}+\sqrt{r_{1}^{2} q^{\prime \prime}}\left[(\Delta q)^{2}-3 \Omega\left(\Omega-\Omega_{* p i}\right)\left(\frac{\bar{\omega}_{d m}^{2}}{\omega_{A}^{2}}\right)\right]^{1 / 2}[\Delta q+ \\
& \left.\left((\Delta q)^{2}-3 \Omega\left(\Omega-\Omega_{* p i}\right) \frac{\bar{\omega}_{d m}^{2}}{\omega_{A}^{2}}\right)^{1 / 2}\right]^{1 / 2}+\left\langle\beta_{h} \hat{I_{0}}\right\rangle \Omega \log \left(1-\frac{1}{\Omega}\right)=0
\end{aligned}
$$

where $\Omega=\omega / \bar{\omega}_{d m}, \bar{\omega}_{d m}=\bar{\omega}_{d h}\left(E=E_{m}\right), \bar{\omega}_{d h}$ is the toroidal precession frequency of the trapped energetic particles, $E_{m}$ is the upper bound of the energy range of the fast ion distribution, $\delta \hat{W}_{f}=2 R_{0} \delta W^{M H D} / \pi r_{1}^{2} B_{0}^{2}\left|\xi_{0}\right|^{2}, \delta W^{M H D}$, is given in references [21] 

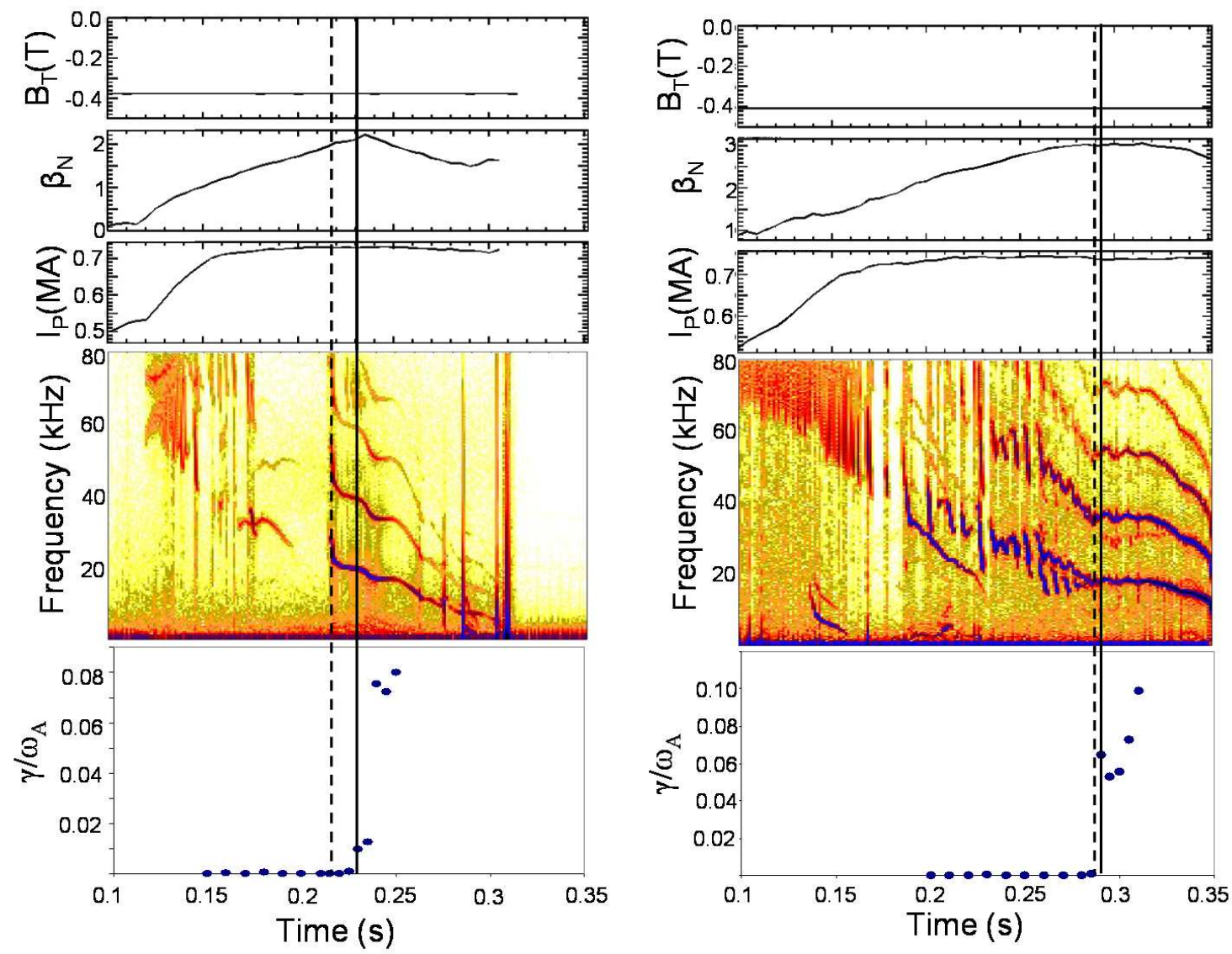

Figure 3. The toroidal field, $\beta_{N}$, plasma current, mode spectrogram and growth rate of the internal $n=1$ mode found from linear MHD stability analysis for MAST discharges (left) 21792 and (right) 22073. The dashed vertical line shows the experimentall observed mode onset time and the solid line shows that predicted numerically, in good agreement for two different MAST plasmas.

and [27], $\beta_{h}$ is the fast ion beta, the Alfvén frequency is $\omega_{A}=v_{A} / R, v_{A}=B_{0} / \sqrt{\mu_{0} \rho_{0}\left(r_{1}\right)}$, $\Omega_{* p i}$ is the ion diamagnetic frequency and $\hat{I}_{0}$ is defined in reference [26]. For fishbones to be unstable in plasmas with monotonic $q$-profiles, the drive from the fast ion pressure must be sufficiently large as to overcome the Alfvén continuum damping around the $q=1$ surface. However, the continuum damping can be eliminated in reversed shear plasmas without the $\omega_{* p i}$ diamagnetic terms to produce a continuum gap. Indeed when $\Delta q>0$, the continuum gap is in the range $\left[-\Delta q \omega_{A} / \sqrt{3},+\Delta q \omega_{A} / \sqrt{3}\right]$.

It is possible to compare the $\Delta q$ required to drive fishbones compared to that required for the ideal internal mode to become unstable. Figure 5 shows the $\Delta q$ at which the ideal internal kink becomes unstable is lower than that at which $n=1$ fishbones can be driven unstable for a circular large aspect-ratio plasma with a slowingdown distribution as stated above. There are two unstable branches, one ideally fluid driven and one driven by the fast ions. The ideal internal pressure-driven branch is most unstable when $\Delta q$ is less than a critical threshold. Above this the fishbone fast-ion driven branch is unstable, with a critical $\Delta q$ determined by the fast ion beta. 


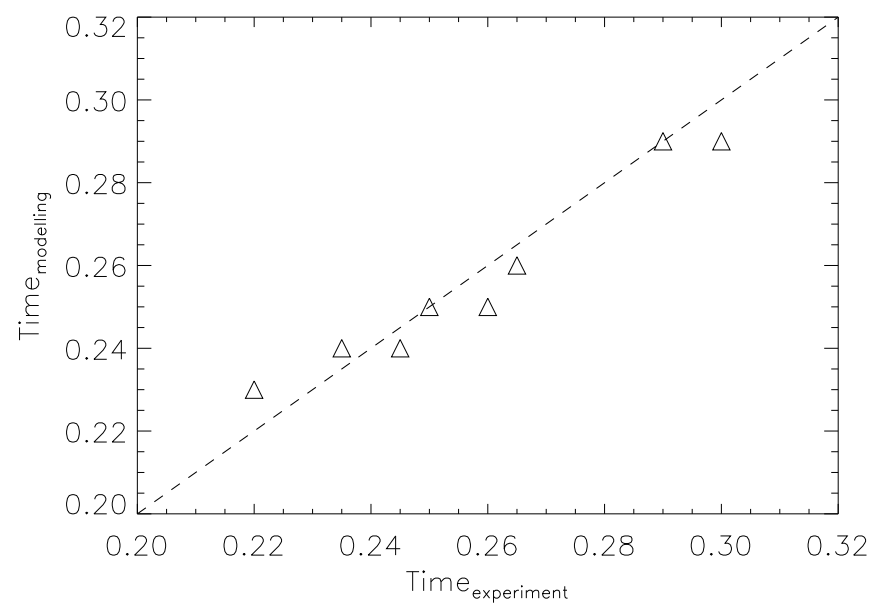

Figure 4. The experimentally observed time of LLM onset with respect to the time of reconstruction of the plasma equilibrium when the ideal $n=1$ is first found to be unstable, showing excellent agreement between empirical observation and ideal MHD predictions.
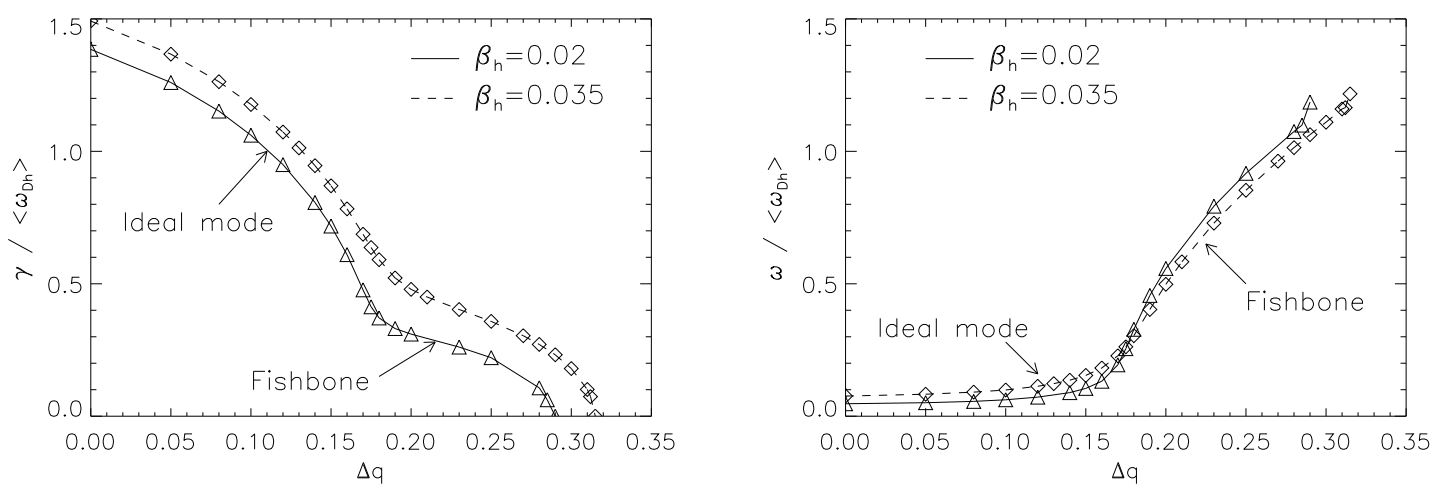

Figure 5. The (left) growth rate and (right) frequency of an $n=1$ mode with respect to $\Delta q$. For small enough $\Delta q$ the ideal fluid-driven mode dominates, but above a critical threshold of $\Delta q \sim 0.17$, the fast-ion driven fishbone is unstable

The fact that fishbones would be unstable at a larger $\Delta q$ than required for the LLM to become unstable agrees well with the behaviour exhibited experimentally as the safety factor evolves. Once $\Delta q<\Delta q_{\text {crit }}$ and the LLM becomes unstable, which in turn redistributes the fast ions such that there is no longer sufficient gradient in either radial or velocity space of the energetic ion distribution within the core to drive the fishbones, and the chirping activity ceases. It is also interesting to note that the fishbones are significantly more unstable in non-monotonic $q$-profile plasmas, since the drive does not have to overcome the Alfvén continuum damping. Figure 6 shows the growth rate of the fishbones with respect to the fast ion pressure for two cases, one with a monotonic $q$-profile with $\Delta q=-0.25$ and one with a reversed-shear $q$-profile with $\Delta q=0.25$, with 


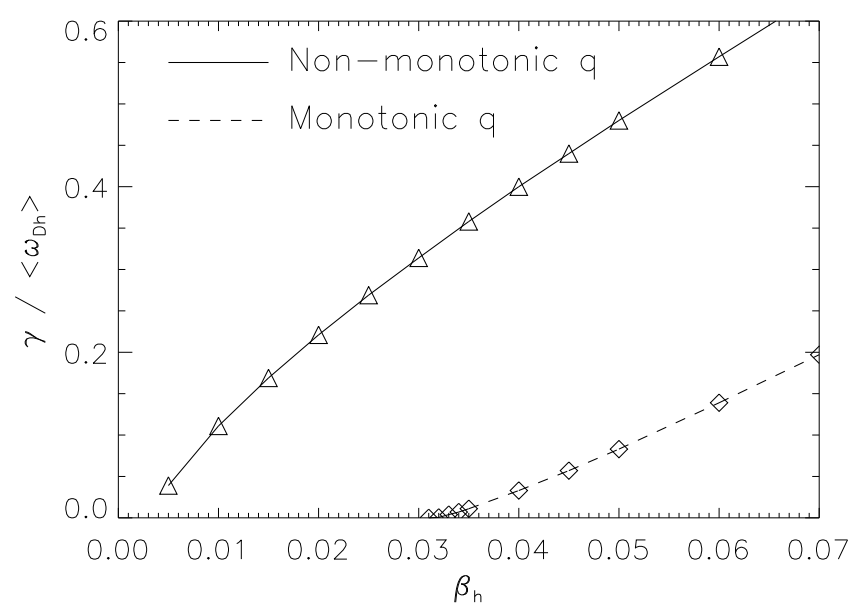

Figure 6. The growth rate of an $n=1$ fishbone mode with respect to $\beta_{h}$ for both a monotonic and a non-monotonic $q$-profile with $|\Delta q|=0.25$.

$\left\langle\omega_{d h}\right\rangle / \omega_{A}=0.1$ and $\omega_{A} /\left\langle\omega_{d h}\right\rangle \cdot \epsilon_{1}^{2} \pi \delta W_{T}=+1,-1$ in the non-monotonic and monotonic cases respectively, which enters into the term for $\delta W_{f}$ in equation 2. This explains why chirping activity is observed in MAST plasmas with large $\Delta q$.

\section{Rotation braking compared to neoclassical toroidal viscosity predictions}

Plasma rotation in tokamaks can have a stabilizing effect on both micro- and macroinstabilities, leading to increased confinement. In MAST, the neutral beam injection can lead to a core plasma velocity $\sim 400 \mathrm{~km} / \mathrm{s}$ (Alfvén Mach number $\sim 0.3$ ). Following the onset of the LLM, the plasma core rotation is rapidly damped whilst the mode's rotation frequency remains constant. In previous MAST experiments, Neoclassical Toroidal Viscosity (NTV) theory [29], arising from the non-axisymmetry of the magnetic field, has been shown to provide an appropriate mechanism for the fast, distributed braking that is observed experimentally in the presence of the LLM $[20,31]$.

Recently, external magnetic perturbations have also been applied in co- and counterNBI heated plasmas to further test NTV theory. Non-axisymmetric $n=2$ fields have been applied from MAST's ex-vessel coils - consisting of four coils each spanning $83^{\circ}$ toroidally and containing three turns, with each turn capable of carrying $5 \mathrm{kA}-$ and braking measured with charge exchange recombination spectroscopy. The damping observed in a counter-NBI plasma is illustrated in figure 7. A very clear global braking is observed, with the rotation reducing by a factor of two in approximately $50 \mathrm{~ms}$. There are no magnetic island or ideal instabilities present in the plasma to induce torques on the plasma, so this effect is from the applied external field. This experimental braking has been compared to the predicted torque arising from NTV theory. There is a factor of five discrepancy between experiment and theory, though by reducing the resonant component for two harmonics around a given resonant surface by a factor 


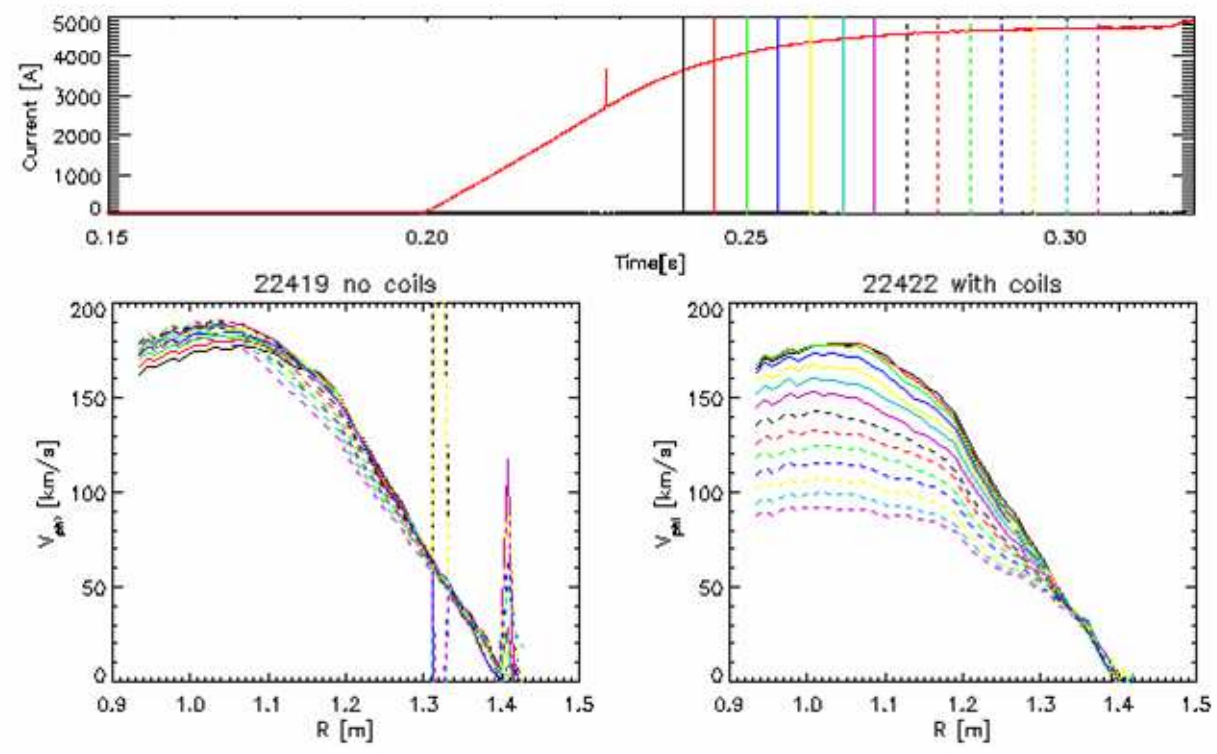

Figure 7. The rotation profile in MAST shot 22422 exhibiting strong braking when an $n=2$ external field is applied

of 0.5 (where 1 is complete plasma shielding) the agreement is improved, suggesting a strong plasma shielding of the resonant torque. Further modelling to include the plasma response rigorously (which is implicitly included in the simulations of braking from the LLM) will be undertaken with MARS [32]. Future experiments will attempt to develop MHD-quiescent comparison co-NBI plasmas in order to assess the neoclassical offset term expected from theory and scan ion collisionality to test NTV theory in lower collisionality regimes relevant to ITER.

\section{Measuring temperature profiles around transient MHD events}

Performance is often limited in high $\beta$ plasmas by the occurrence of neoclassical tearing modes (NTMs) [16]. In MAST, many NTMs occur after being seeded by a sawtooth crash. Consequently, it is important to understand the physical mechanisms that underlie the sawtooth crash with a view to understanding how this can act as a seed for NTMs and how to avoid this in future devices. The upgraded MAST Thomson Scattering (TS) system provides excellent spatial resolution $(\sim 1 \mathrm{~cm})$ at over 130 radial locations across the full plasma diameter, and utilises 8 individual lasers which can be operated in burst mode, whereby the laser separation can be adjusted to within a few microseconds. This provides detailed profiles of transient and periodic phenomena such as temperature perturbations associated with sawteeth.

The evolution of the electron temperature profile has been captured every $20 \mu \mathrm{s}$ through a sawtooth crash, as described in reference [33]. An $m=n=1$ magnetic island grows rapidly leading to a strong increase in the electron temperature gradient at the island boundary layer. The island width grows and the increasing temperature 


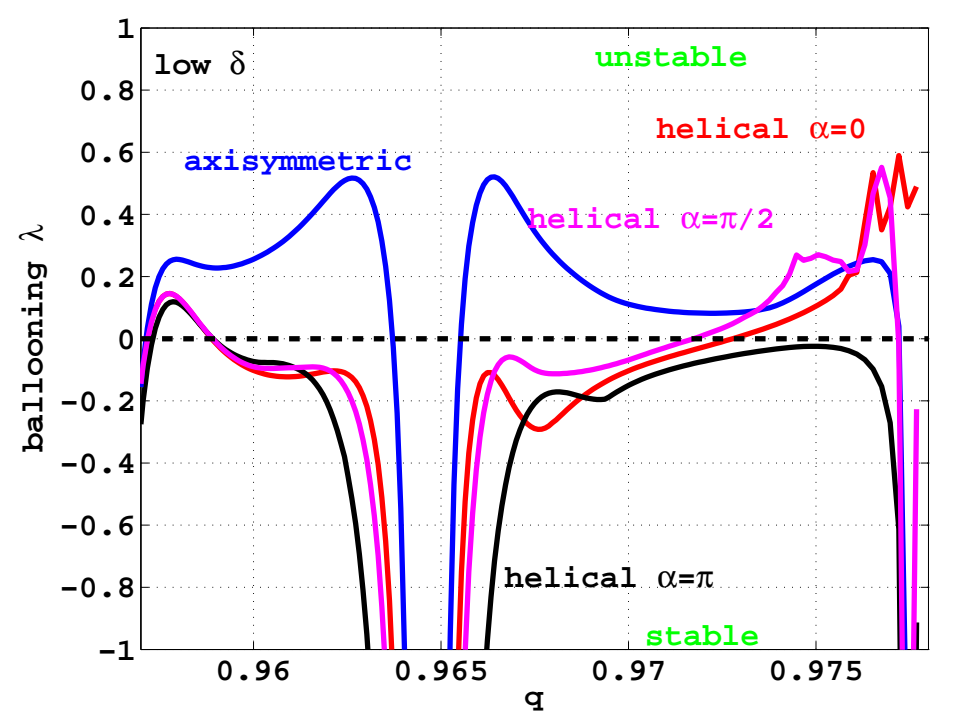

Figure 8. The infinite- $n$ ballooning mode growth rate $(\lambda>0$ is unstable) of MAST discharge 24479 as a function of $q$ for both $2 \mathrm{~d}$ and $3 \mathrm{~d}$ treatment of the plasma core at $t=333.41 \mathrm{~ms}$. In the $3 \mathrm{~d}$ case, the stability is shown for different toroidal angles at which the field line crosses the outboard side of a flux surface, $\alpha$. Since the $3 \mathrm{~d}$ treatment allows for the clustering of flux surfaces on the low field side due to the perturbation, ballooning modes become more stable in the core, but are more unstable at the boundary layer.

gradient moves into regions of lower magnetic shear, before the sawtooth crash occurs in less than $20 \mu \mathrm{s}$. During this island evolution, over the course of $100 \mu \mathrm{s}$, the electron density does not change and the safety factor stays below unity throughout the cycle. The non-axisymmetric plasma in the presence of a growing magnetic island is found to be unstable to interchange or ballooning modes, which are postulated to result in the rapid crash following instability growth on Alfvénic timescales. This supports the hypothesis that the sawtooth occurs due to a pressure-driven magnetohydrodynamic instability triggered by an increase in the electron temperature gradient arising from magnetic reconnection [34-36].

Non-axisymmetric stability analysis of the kinked equilibrium that results when a very large magnetic island exists (island width $\sim 30 \mathrm{~cm}$ compared to a minor radius $\sim 55 \mathrm{~cm}$ ) has been undertaken using the VMEC $3 \mathrm{~d}$ equilibrium code [37] and the TERPSICHORE 3d linear stability code [38]. The toroidal variation of the perturbation is taken from an equilibrium which spontaneously develops a bifurcated helical core structure [39], whilst the $q$-profile is obtained from EFIT equilibrium reconstruction constrained by MSE measurements made shortly before the crash and the pressure gradient is taken from TS measurements. During the island evolution the plasma core is assumed to be incompressible, so the enclosed flux in the area of the hot core is the same as before the kink perturbation, implying that a current sheet arises at the boundary layer between the core plasma which has $q<1$ and the separatrix 
where $q=1$. This assumption allows the safety factor at the boundary layer to be inferred from the pre-island equilibrium. The core plasma shape is taken from the $m=n=1$ perturbation found by axisymmetric linear stability analysis. When the helical perturbation is included, the plasma core becomes stable to ballooning modes except near the boundary layer where instability is exacerbated since the $3 \mathrm{~d}$ deformation tends to squeeze the pressure gradient outwards. The infinite- $n$ ballooning stability is illustrated in figure 8 for both an axisymmetric core plasma and in the case where the full $3 \mathrm{~d}$ helical perturbation is modelled. In the $3 \mathrm{~d}$ case, the stability is shown for different toroidal angles at which the field line crosses the outboard side of a flux surface, $\alpha$. When the helical perturbation is modelled, the pressure gradient tends to get squeezed towards the edge of the radial domain. The ballooning stability is found to be worse on field lines that cross the outer edge of the plasma in the region $-\pi / 2<\phi<\pi / 2$ where the kink perturbation causes pressure squeezing in a region of unfavourable curvature.

\section{Resistive Wall Modes}

If NTMs and LLMs can be avoided, the ultimate performance limit in advanced tokamak scenario operation is set by the resistive wall mode (RWM). In the absence of a surrounding wall, the plasma is stable to kink modes until the normalised plasma pressure exceeds a critical value, $\beta_{\infty}$. In the presence of an ideally conducting wall, the plasma is stable to a critical value, $\beta_{b}$, with the range $\beta_{\infty}<\beta<\beta_{b}$ called the wallstabilised region. In practice, since the vessel wall has a finite resistivity, the magnetic perturbation of the external kink mode can penetrate the wall on the time scale required for eddy currents to decay resistively and so wall-stabilisation is degraded. Operation above the no-wall beta limit is important in advanced tokamak regimes, since these plasmas are susceptible to the pressure-driven kink mode due to broad current profiles and the associated low no-wall beta limits.

\subsection{Resonant Field Amplification}

A weakly damped mode, such as the RWM in the presence of rotation or kinetic damping, can amplify the resonant component of magnetic field asymmetries [40]. One way to probe RWM stability is to examine the plasma response to externally applied non-axisymmetric magnetic fields. When the plasma pressure exceeds the no-wall $\beta$ limit, strong resonant field amplification (RFA) occurs. The resonant field amplification amplitude is usually defined as the ratio of the plasma response to the externally applied field, $B_{r}^{\text {ext }}$. It can be found by measuring the perturbed radial magnetic field at the wall, $B_{r}$, as

$$
A_{\mathrm{RFA}}=\frac{B_{r}\left(r=r_{\text {sensor }}\right)-B_{r}^{\text {ext }}}{B_{r}^{\text {ext }}}
$$

$A_{R F A}$, is a complex number, whose phase angle refers to the toroidal phase of the plasma response with respect to the externally applied field [41]. 


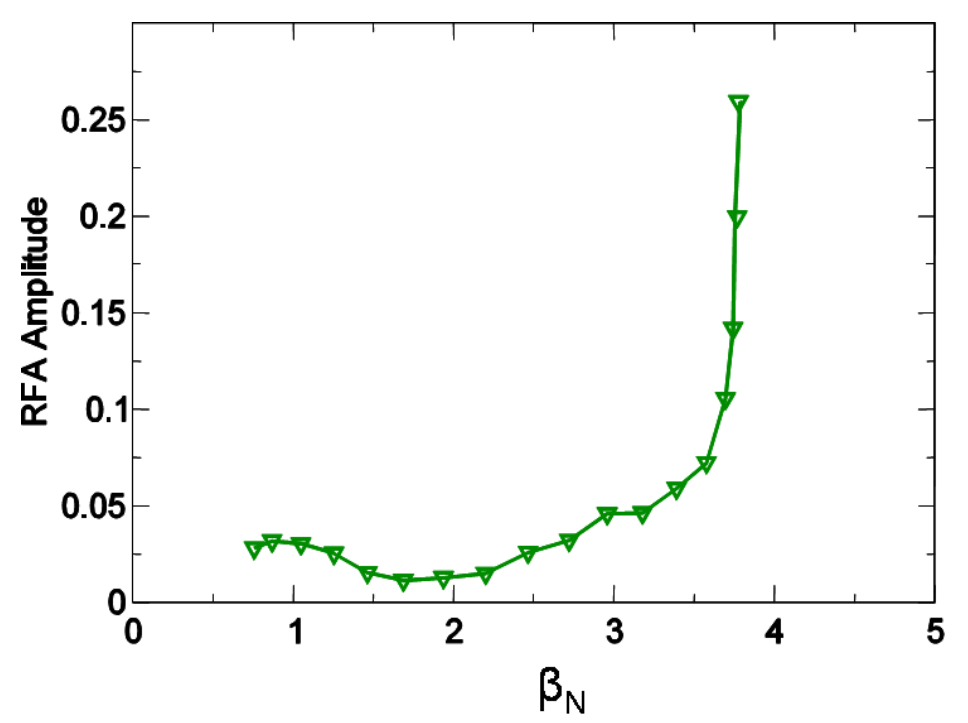

Figure 9. The amplitude of the resonant field amplification with $60 \mathrm{~Hz}$ applied field in MAST shot 25109 with respect to normalised pressure, showing a clear increase in the RFA after $\beta_{N}=3.5$, compared to a no-wall ideal stability limit predicted to be $\beta_{N}=4$.

In MAST, oscillating non-axisymmetric magnetic fields have been applied from the internal coils, consisting of two rows of six coils, equally spaced toroidally and located symmetrically above and below the midplane and mounted inside the vacuum vessel. The coils are made of four turns and can take up to $1.4 \mathrm{kA}$ in each turn. In these experiments, toroidally opposite coils are connected in series with oppositely directed currents to produce an $n=1$ field. The external field is applied in H-mode plasmas which reach $\beta_{N} \sim 5.5$, and exceed the ideal no-wall MHD stability limit predicted by MishKA-1 [42] stability analysis. The external field is applied in identical plasma discharges with frequency varying from $30 \mathrm{~Hz}$ to $100 \mathrm{~Hz}$ rotating in the same direction as the plasma rotation. During the discharges the neutral beam heating power is increased and the plasma current is ramped down from $600 \mathrm{kA}$ to $500 \mathrm{kA}$ in order to progressively increase $\beta_{N}$, allowing the plasma response to be measured for different values of plasma pressure. By measuring the plasma response on pairs of saddle coils, both in the same sectors of the vessel as the internal coils from which the magnetic perturbation is applied, and also in sectors orthogonal to the applied field, the amplitude of the RFA can be found according to equation $3 . B_{r}^{\text {ext }}$ was measured in a series of vacuum only discharges, and the saddle coil data is corrected by subtracting the signal from a plasma with no applied field in order to remove direct pick up from the poloidal field coils.

Figure 9 shows the RFA as a function of $\beta_{N}$ as the auxilliary power is increased and then the plasma current is reduced in order to further enhance $\beta_{N}$. A clear increase in the RFA is observed at $\beta_{N}=3.5$ in reasonably good agreement with the ideal no-wall limit predicted by the MishkA-1 stability code, where $\beta_{N}^{\infty}=4.0$. The optimal frequency for plasma response to the applied fields is found to be approximately $60 \mathrm{~Hz}$ and so plasma 
parameters can be varied with small oscillating fields applied at this frequency in order to parasitically probe stability. Following this successful demonstration, RFA will be used routinely as a spectroscopic diagnostic of ideal no-wall stability in MAST plasmas. Results from JET [43] and DIII-D [44] also find the RFA limit is often below the ideal nowall limit, leading to a redefinition of the RFA limit as the $\beta_{N}$ at which the logarithmic derivative of the RFA is maximised [45]. However, oscillations in the MAST data mean that the logarithmic derivative of the RFA does not have a clear maximum, so cannot be used to infer the experimental no-wall stability limit. Nonetheless, the qualitative agreement between the RFA measurements and the linear stability analysis, coupled with similar discrepancies between the two limits in other devices, gives reasonable confidence that the modelling is an accurate representation of the $\beta$-limits in these MAST plasmas.

\subsection{Modelling RWM Stability}

Various experiments have shown that the plasma can operate above the no-wall beta limit in the presence of rotation [46-51]. Recent experiments with nearly balanced NBI [54-56] have found a critical velocity to stabilise the RWM well below that found in the magnetic braking experiments [50,52]. Furthermore, preliminary results from NSTX suggest that operation above the no-wall limit can be attained even with no plasma rotation at the resonant $q=2$ surface [57]. Consequently, there is a need to understand the passive stabilisation occurring from kinetic effects which is thought to underlie these results, especially given that the rotation in ITER is uncertain and predicted to be relatively low [58]. Various models have been presented to explain the RWM damping due to kinetic effects arising from particle motion. For instance, RWM stabilisation has been attributed to sound-wave damping [59], ion Landau damping [60], or more recently, precessional drift resonance with the trapped thermal ions $[61,62]$. Both thermal and fast ion damping effects have since been assessed numerically [63-68].

In order to assess the rôle of these kinetic effects in damping the RWM in MAST, the drift-kinetic particle-following HAGIS code [71] has been used to calculate the change in the potential energy of the kink mode in the presence of both trapped and passing ions. The code can be used in a perturbative manner to study kinetic resonance damping effects by diagnosing which particles cause the strongest wave-particle interactions. Whilst this gives an exact treatment of the kinetic effects by calculating the orbit frequencies of a distribution of markers, it does not self-consistently evolve the eigenmode structure in the presence of the particles, which has been shown to affect the RWM stability [64]. HAGIS is a particle-orbit code following the guiding centre motion of the particles, meaning that all the relevant resonances with particle motion are included rigorously. This modelling includes finite drift orbit width effects that have been neglected in previous studies [62,64-68].

The change in the potential energy of the unstable mode due to kinetic effects is 
expressed as [69]

$$
\delta W_{K}=-\frac{1}{2} \int \xi_{\perp}^{*} \cdot\left(\nabla \cdot \tilde{\mathbb{P}}_{K}\right) \mathrm{d} V
$$

where $\xi$ is the plasma displacement eigenfunction, $\tilde{\mathbb{P}}_{K}$ is the perturbed pressure tensor which can be found by taking moments of the perturbed distribution function, which itself can be found by solving the linearised drift kinetic equation, and $V$ is phase space including both velocity and volume integrals. By solving the drift kinetic equation and substituting in for $\tilde{\mathbb{P}}_{K}$ then performing phase space integrals over particle energy, $\mathcal{E}$, pitch angle, $\lambda=v_{\|} / v$, and flux, $\psi$, it can be shown that the kinetic potential energy of the mode incorporates a frequency resonance condition $[61,68,70]$,

$$
\delta W_{K} \sim \sum_{l=-\infty}^{\infty} \frac{\left(\omega-n \omega_{E}\right) \partial f_{j} / \partial \mathcal{E}-\frac{1}{e Z_{j}} \partial f_{j} / \partial \psi}{\omega-n \omega_{E}+i \nu_{e f f, j}-\langle\omega\rangle_{d, j}-l \omega_{b, j}}
$$

where $\omega$ is the complex eigenmode frequency, the $E \times B$ frequency is $\omega_{E}=\omega_{\phi}-\omega_{* i}$, $\omega_{\phi}$ is the toroidal rotation frequency, $\omega_{* i}$ is the ion diamagnetic frequency, $Z$ is the effective plasma charge, $e$ is the electron charge, the particle energy is $\mathcal{E}=v^{2} / 2$, $\langle\omega\rangle_{d}$ is the orbit-averaged drift precession frequency, $\omega_{b}$ is the trapped-particle bounce frequency and $\nu_{\text {eff }}$ is the collision frequency. Using the HAGIS code, all of the toroidal and poloidal bounce(transit)-averaged frequencies for any species of particles can be calculated, though collisions are neglected. It is clear from equation 5 that a resonance between the Doppler shifted mode frequency and these particle frequencies can occur when the denominator vanishes. Whilst it has been shown that the low-frequency RWM can resonate with both the precession drift frequency and bounce frequencies of thermal ions (depending on the plasma rotation) $[63,65,66]$, for energetic particles typically $\omega_{d}, \omega_{b} \gg \omega-\omega_{E}$. Consequently, it is likely that the contribution from the energetic particles is not a resonant process, but related to (i) an increase in the resistance to changes in the magnetic flux by the kink mode through an inhibition of toroidal coupling of harmonics and (ii) finite orbit width effects of the energetic ions.

In order to simulate the importance of finite orbit width effects on RWM stability, the effect of changing $B_{t}$ on the change in the mode potential energy due to the fast ion kinetic contribution, $\delta W_{h}$, has been considered. The radial excursion of energetic ion orbits can be expressed as [72]

$$
\Delta_{r}=\frac{q M}{r e Z B_{0}}\left(\left|v_{\|} R-l R_{0}^{2} q \omega_{b}\right|\right)
$$

where $M$ is the ion mass, $e$ is the electron charge, $Z$ is the effective plasma charge, $v_{\|}$ is the velocity of the ions parallel to the magnetic field, $R\left(R_{0}\right)$ is the major radius (at the magnetic axis), $l=0$ for trapped ions and $l=1$ for passing ions and $\omega_{b}$ is the bounce frequency of the ions. Evidently, increasing $B_{0}$ will decrease the orbit width of the particles. Figure 10 shows $\Re e\left(\delta W_{k}\right)$ calculated using a drift kinetic code for a typical MAST NBI fast ion distribution as the toroidal magnetic field is varied. The 


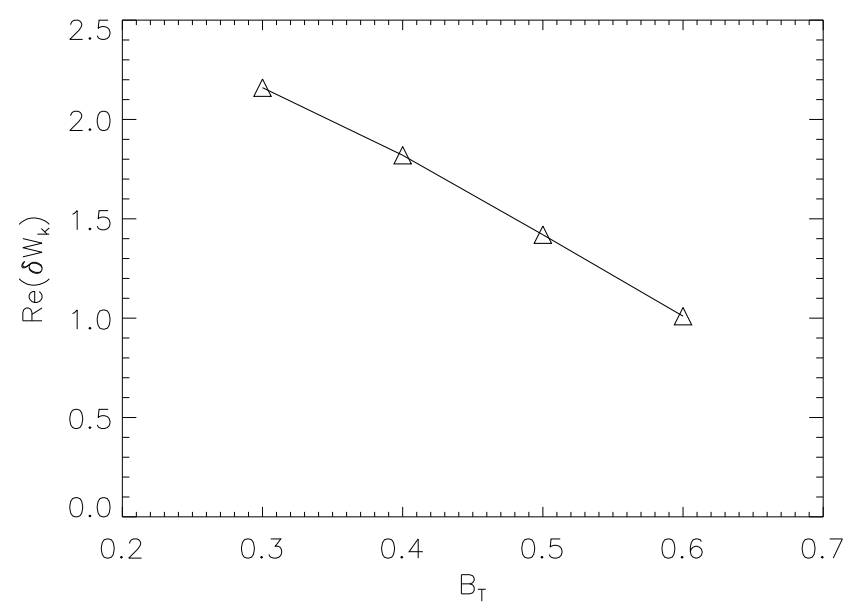

Figure 10. The change in the potential energy of the external kink mode arising due to the presence of energetic particles as a function of the toroidal magnetic field. As $B_{t}$ is increased, and so the radial excursion of the ion orbits decreases, the effect of the fast ions is diminished.

growth rate of the RWM can be formulated in terms of the MHD perturbed energy and the change due to kinetic corrections as [62]

$$
\gamma \tau_{W}=-\frac{\delta W_{\infty}+\delta W_{K}}{\delta W_{b}+\delta W_{K}}
$$

where $\delta W_{b, \infty}$ represents the sum of the plasma and vacuum energy with and without a wall respectively and where $\delta W_{\infty}<0$ and $\delta W_{b}>0$, indicating that the plasma is in the region where the ideal external kink mode is stable, but the RWM is MHD unstable. Thus, it is clear that as the radial excursion of the fast ion orbits decreases (with increasing magnetic field), the effect of the fast ions on external kink stability is diminished.

\subsection{Modelling the nonlinear interaction of RWMs with other MHD instabilities}

The JoREK code $[73,74]$ has been used to explicate the nonlinear interplay between the RWM and other plasma instabilities. The nonlinear behaviour of the RWM has been studied in general toroidal geometry by solving the reduced MHD equations. The hot core plasma, with density $\rho_{0}$ is surrounded by a cold plasma with low density, $\rho_{w}$, and high resistivity. Outside the cold plasma is a resistive wall at $r=r_{r w}>a$ with resistivity $\eta_{w}$, which in turn is surrounded by a vacuum and an ideal wall at $r=r_{w}>r_{r w}$. Figure 11 shows that whilst the linear growth rate of the RWM for $r_{r w}=1.3 a$ depends upon the density of the cold temperature, high resistivity plasma surrounding the core plasma, the nonlinear saturation level does not, in good agreement with cylindrical modelling $[75,76]$. If $\rho_{w} / \rho_{0}$ is dropped below $10^{-3}$, the linear growth rate does not change, and is governed by the inverse wall time. JoREK simulations also show that when the resistive wall is 


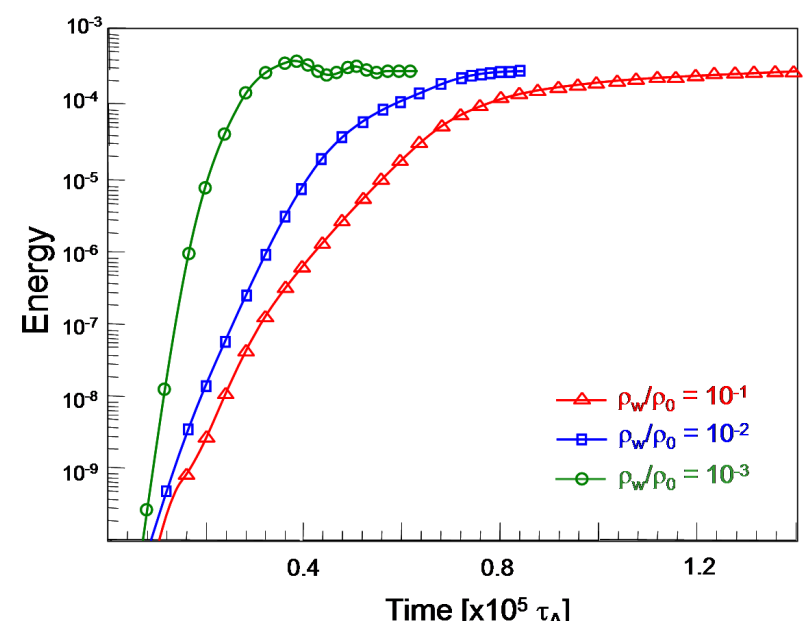

Figure 11. Time evolution of the magnetic energy of the RWM for different $\rho_{w}$.
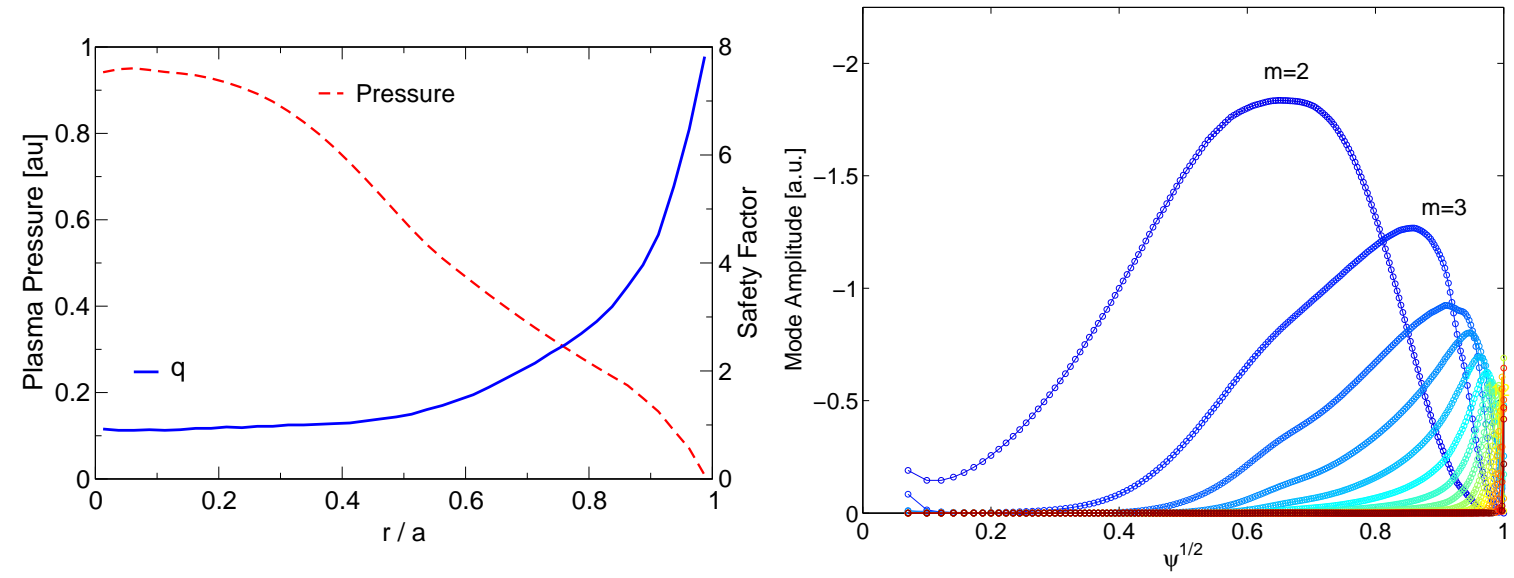

Figure 12. (left) The safety factor and pressure profiles for a typical MAST discharge used to simulate the nonlinear evolution of RWMs. (right) The linear eigenstructure of the RWM.

close to the plasma, $r_{r w}<1.2 a$, neither the linear growth rate nor the energy saturation level of the RWM varies with $\rho_{0} / \rho_{w}$.

Whilst the RWM has not been observed in MAST, nonlinear simulations of RWMs have also been performed for typical MAST profiles at artificially inflated plasma pressure such that the equilibrium is found to be unstable to both the $m / n=2 / 1$ RWM and an internal 3/2 mode. The safety factor and pressure profiles are illustrated in figure 12 together with the eigenfunction of the linear 2/1 RWM.

Figure 13 illustrates that in the presence of the RWM, the $3 / 2$ mode is significantly destabilised in the nonlinear phase, due to the change in current profile at the $q=3 / 2$ surface resulting from the growth of the $n=1$ mode. The dominant poloidal harmonic is found by examining the perturbation in the poloidal plane. This example illustrates the nonlinear mode coupling which can be studied with JOREK, even using reduced MHD which neglects some important tearing mode physics. Such interaction between 


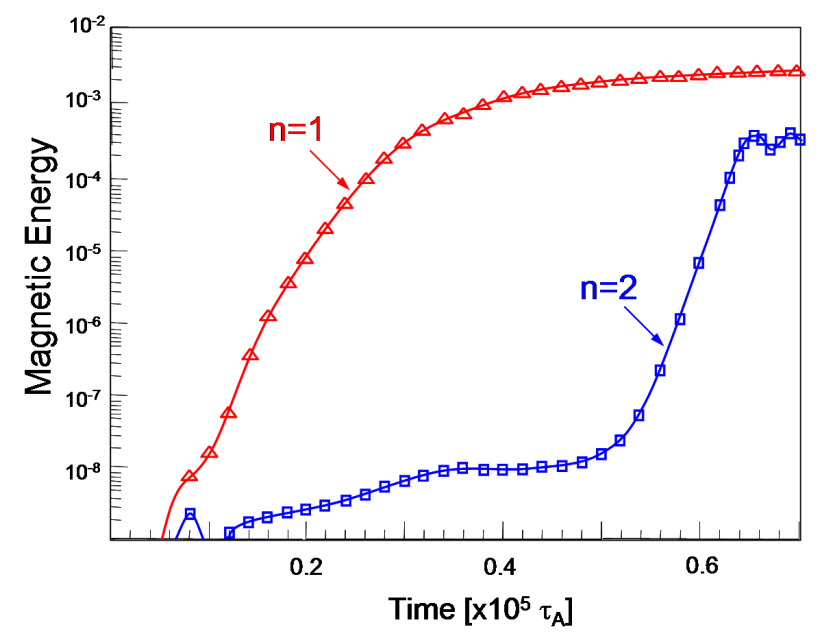

Figure 13. Time evolution of the magnetic energy of the $3 / 2$ and $2 / 1$ components. The $3 / 2$ mode is destabilised in the nonlinear phase.

the RWM and other core MHD instabilities may help to shed light on recent observation of RWM coupling with energetic particle driven modes [77,78] and ELMs [79].

\section{Stability analysis of plasmas following an upgrade to MAST}

The excellent agreement between stability limits for the internal ideal $n=1$ mode and the experimental observation of an ideal saturated long-lived mode shown in section 2 and the characterisation of the no-wall limit using resonant field amplification and advances in numerical simulation of RWM stability discussed in section 5, allows us to utilise these numerical methods to study the stability of plasmas foreseen in an upgraded MAST [80]. The effects of both rapid rotation and significant fast particle populations have been included in the stability simulations of both the infernal mode and the RWM in order to determine the expected operating space in the new device. The presence of both on- and off-axis neutral beam heating following the upgrade makes it possible to experimentally investigate a range of important stability issues not presently accessible. The new current drive capabilities enable the generation of CTF-like $q$ profiles $[81,82]$ in which $q_{\min }>1.5$ and allow the stability limit of these scenarios to be investigated. MAST Upgrade stability studies will aim to demonstrate reliable operation at high $\beta_{N}$ by understanding the characteristics and impact of performance-limiting instabilities in order to confidently extrapolate performance to next-step devices like an ST-CTF. The upgrade will facilitate this by allowing greater flexibility in $q$-profile, fast ion distribution, density (and hence collisionality), shaping and rotation profile which all strongly determine infernal mode and RWM stability.

At CTF relevant normalised pressures of $\beta_{N} \sim 3.5$, the limiting MHD phenomena are expected to be internal modes, with resistive wall modes playing little role. Off-axis NBCD will allow quantification of the benefit of operation at elevated safety factor in 
Figure 14. The safety factor and pressure profiles for the fully non-inductive longpulse scenario in MAST Upgrade as the fast ion diffusion rate is increased.

order to optimise the operating scenario for CTF. At the same time, by operating at high current $(2 \mathrm{MA})$ and high density $\left(2.3 \times 10^{20} \mathrm{~m}^{-3}\right)$, the increased heating capabilities allow access to higher $\beta_{N}$ where external kink modes are expected to limit performance, allowing studies of great importance for ITER advanced scenarios. The installation of passive stabilisation plates widens the stable window between ideal no-wall and withwall limits, giving enhanced scope for improved physics understanding of RWM damping from rotation and kinetic effects.

The accessible operating space is ultimately bounded by ideal stability limits. In reality, resistive instabilities may be expected to play a role before these limits are reached but they nevertheless provide a useful check of the accessibility of the foreseen scenarios. The different plasma scenarios envisaged in MAST Upgrade [80] have been tested to demonstrate ideal stability to low- $n$ modes $(n=1-3)$ at the foreseen operating conditions, both with and without a perfectly-conducting wall (here comprising the poloidal field coil casings and the passive vertical stabilisation plates) and with toroidal rotation profile predicted by TRANSP.

Assuming a moderate amount of additional fast ion diffusion, namely $D_{F I}=$ $0.5 \mathrm{~m}^{2} \mathrm{~s}^{-1}$, all of the scenarios are found to be stable at the target values of $\beta_{N}$. As the level of additional fast ion diffusion is increased however, both the achievable $\beta_{N}$ and the minimum value of the safety factor, $q_{\min }$, decrease. As $q_{\min }$ approaches unity, the scenarios become unstable to $n=1$ internal modes and neither the presence of an ideally conducting wall, nor plasma flow, can completely suppress the mode. Such internal modes are often observed in present MAST plasmas, as discussed in section 2. Avoiding such deleterious instabilities is paramount in an ST-CTF, and is one of the major motivations behind the inclusion of off-axis NBI in the upgrade. 


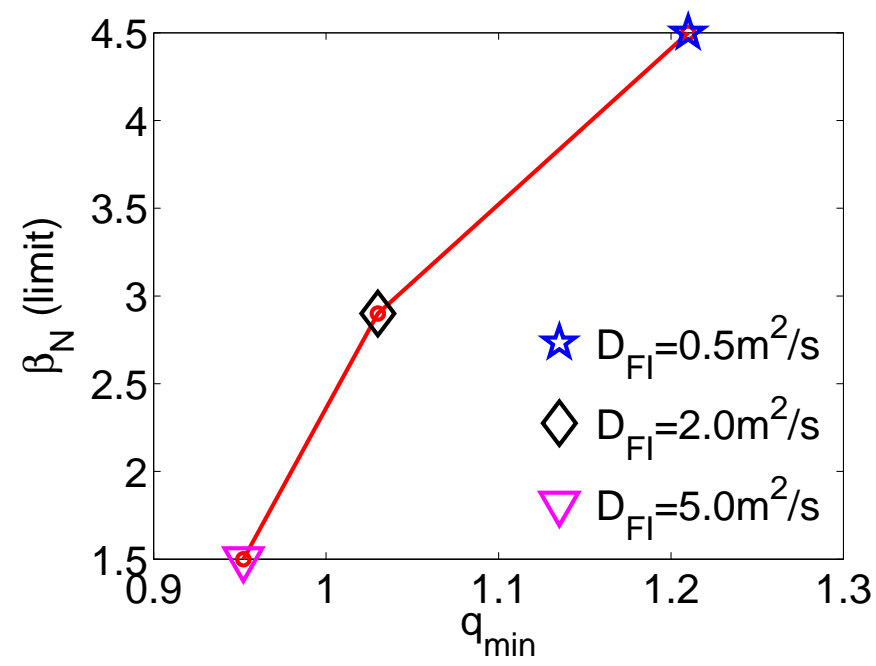

Figure 15. Plot showing change in stability limit $\left(\beta_{N}\right)$ as a function of $q_{m i n}$.

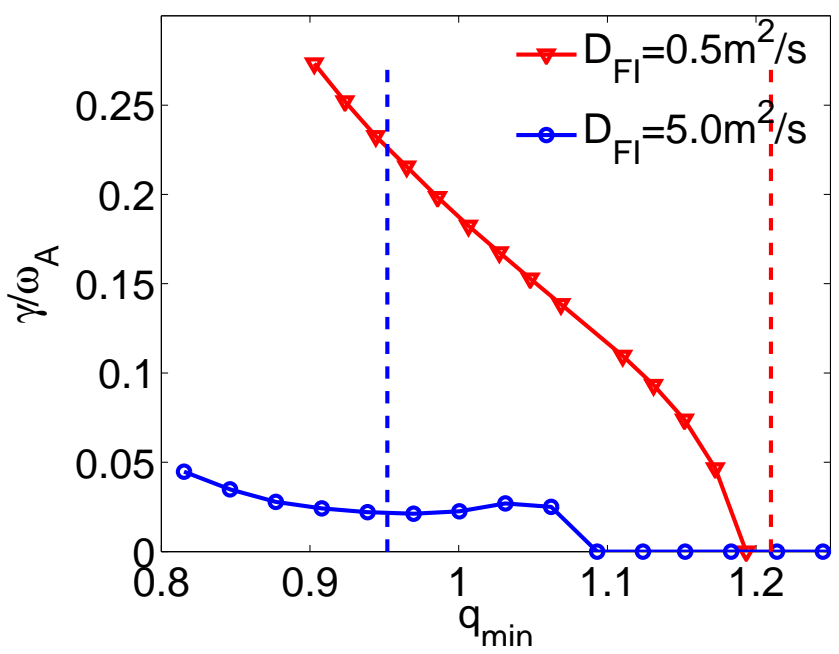

Figure 16. The growth rate of the limiting $n=1$ internal mode as a function of $q_{\text {min }}$ as the toroidal field is scanned. The additional fast ion diffusion is assumed to be $D_{F I}=0.5 \mathrm{~m}^{2} \mathrm{~s}^{-1}$ or $D_{F I}=5 \mathrm{~m}^{2} \mathrm{~s}^{-1}$.

In cases where $q_{\min }$ falls below unity, sawtooth oscillations are expected to occur. This is often the key limitation to performance and pulse-length in present MAST plasmas as the first sawtooth, which is usually lengthened by a significant population of fast ions inside $q=1$, can be disruptive. However, the new NBI geometry will lead to very different plasma rotation profiles and fast ion distributions, which dominate the sawtooth behaviour [83,84]. Consequently, experiments have been conducted with offaxis NBI by vertically displacing the plasma. It was found that the sawtooth amplitude drops and the frequency increases as the NBI fast ion population is peaked progressively 
further outside the $q=1$ surface [85]. Consequently, the sawteeth in the baseline scenarios with $q<1$ are likely to be smaller and more frequent due to the off-axis beam ion deposition outside the $q=1$ radius. These more benign sawteeth are expected to modify the $q$-profile but are not expected to lead to plasma termination.

It is apparent that $q_{\text {min }}$ plays an important role in setting the $\beta_{N}$ limit and that it falls as the fast ion diffusion rate is increased. The safety factor and pressure profiles for a typical MAST Upgrade scenario as the assumed fast ion diffusion rate is increased are illustrated in figure 14, clearly showing that as $D_{F I}$ increases, both $q_{\min }$ and the achievable pressure decrease. Figure 15 presents the relationship between the $\beta_{N}$ limit and $q_{\min }$ for the fully non-inductive long-pulse scenario as the fast ion diffusion rate is scanned up to, and beyond, the stability boundary. The stability of the scenarios is also strongly affected by the assumptions of neutral beam driven current in the transport simulations used to define the scenario profiles. For reference the target operating $q$-profile has $q_{\min }=1.21$ and considers the anomalous fast ion redistribution to be driven by a diffusion coefficient of $D_{F I}=0.5 \mathrm{~m}^{2} \mathrm{~s}^{-1}$. Therefore, it is clear that there is a significant risk that the scenario will become unstable to internal modes if this cannot be sustained due to, for instance, degraded neutral beam current drive or current redistribution from energetic particle modes. Consequently, it may be necessary to reduce the operating current or increase the toroidal field in order to keep the safety factor sufficiently elevated to avoid such internal modes.

The sensitivity of the plasma stability to changes in the $q$-profile has also been tested by scanning the toroidal field to change the minimum safety factor. This strong dependence of the $\beta$-limit on $q_{\min }$ is of significant importance for hybrid-like $q$-profiles, especially in spherical tokamaks where the critical $q_{\min }$ for instability is increased, as discussed in section 2. Figure 16 shows the growth rate of the global $n=1$ internal mode (which is the performance limiting mode in this scenario) for two different cases of additional fast ion diffusion. For the target operating scenario $\left(D_{F I}=0.5 \mathrm{~m}^{2} \mathrm{~s}^{-1}\right)$, the $n=1$ mode becomes unstable as $q_{\text {min }}$ drops below 1.19. For an equilibrium with a higher diffusion coefficient, the low shear region is even broader, and so the critical $q_{\text {min }}$ at which the plasma becomes unstable to global $n=1$ infernal modes decreases.

\section{Conclusions}

Detailed macroscopic stability studies on MAST facilitated by very high resolution diagnostics have shed new light on performance-limiting instabilities in high $\beta$ plasmas. The saturated long-lived mode was diagnosed as an ideal internal mode growing unstable as $q_{\text {min }}$ approaches one. This mode degrades confinement, expels energetic ions and induces rotation damping that is in good accordance with NTV theory, and consequently future STs must keep $\Delta q$ above the critical threshold for mode onset, typically $\Delta q \sim 0.1$. The LLM saturates nonlinearly at a sufficiently large amplitude of the displacement where the stabilising field-line bending term in the ideal MHD equations dominates and prevents further growth. RWM stability limits have been probed by MHD spectroscopy 
and state-of-the-art numerical modelling. It is found that fast ion kinetic damping of the RWM is strongly sensitive to the orbit widths of the trapped fast ions. RWMs have also been found to interact with other MHD instabilities nonlinearly. By verifying numerically predicted stability limits with those observed for the long-lived mode and RFA limits as a proxy for the no-wall limit in MAST plasmas, credence has been given to the application of these models for simulating stability in plasmas in an upgrade to MAST. All of the scenarios anticipated in the upgraded device are found to be stable due to a combination of passive stabilising structures, very fast plasma rotation and kinetic damping, provided that there is not a significant additional fast ion diffusion, for instance from fast ion driven instabilities.

\section{Acknowledgments}

This work was funded partly by the RCUK Energy Programme under grant EP/I501045 and the European Communities under the contract of Association between EURATOM and CCFE. The views and opinions expressed herein do not necessarily reflect those of the European Commission. This work was carried out within the framework of the European Fusion Development Agreement

\section{References}

[1] Maisonnier D et al, 2006 Fus. Eng. Design, 811123

[2] Shimada M et al, 2007 Nucl. Fusion, 47 S1

[3] Campbell DJ et al, 2006 21st IAEA Fusion Energy Conference, Chengdu FT/1-1

[4] Ward DJ, 2010 Plasma Phys Control Fusion 52124033

[5] Ward DJ, Cook I and Knight PJ, 2000 18th IAEA Fusion Energy Conference, Sorrento IAEACN-77-FT/P2-20

[6] Bickerton RJ et al, 1971 Nat. Phys. Sci., 229110

[7] Becoulet A and Hoang GT, 2008 Plasma Phys. Control. Fusion 50124055

[8] Litaudon X et al, 2007 Plasma Phys. Control. Fusion 49 B529

[9] Joffrin E, 2007 Plasma Phys. Control. Fusion 49 B629

[10] Doyle EJ et al, 2006 Plasma Phys. Control. Fusion 48 B39

[11] Taylor TS 1997 Plasma Phys. Control. Fusion 39 B47

[12] Manickam J et al, 1994 Phys. Plasmas 11601

[13] Sips ACC et al, 2005 Plasma Phys. Control. Fusion 47 A19

[14] Joffrin E, 2005 Nucl. Fusion 45626

[15] Manickam J et al, 1987 Nucl. Fusion 271461

[16] Challis C et al 2009 36th EPS Conference on Plasma Physics P5.172 "Stability and confinement optimisation in the range $\mathrm{q} 0=1-3$ at JET" and http://epsppd.epfl.ch/Sofia/start.htm

[17] de Bock MFM et al, 2009 36th EPS Conference on Plasma Physics, Sofia P5.186 "First multi-chord MSE measurements on MAST" http://epsppd.epfl.ch/Sofia/start.htm

[18] Meyer HF et al, 2009 Nucl. Fusion 49104017

[19] Chapman IT et al, 2010 Nucl Fusion 50045007

[20] Hua MD et al 2010 Eur. Phys. Lett. 9055001

[21] Hastie RJ et al., 1987 Phys. Fluids 301756

[22] Avinash, Hastie RJ, Taylor JB and Cowley SC, 1987 Phys. Rev. Lett. 592647

[23] Wahlberg C and Graves JP 2007 Phys. Plasmas 14110703 
[24] Huysmans GTA et al, 1991 Proc CP90 Conf on Comp Phys p.371

[25] Kerner W et al, 1991 Contr. Fusion and Plas. Phys. 18th Conf. Berlin Part IV p.89

[26] Chen L, White RB and Rosenbluth MN, 1984 Phys. Rev. Lett. 521122

[27] Bussac MN et al, 1975 Phys. Rev. Lett. 351638

[28] Taylor JB, 2003 Phys. Rev. Lett. 91115002

[29] Shaing KC et al, 2003 Phys. Plasmas 101443

[30] Cole AJ et al, 2007 Phys. Rev. Lett. 99065001

[31] Hua MD et al 2010 Plasma Phys Control Fusion 52035009

[32] Liu YQ et al 2000 Phys. Plasmas 73681

[33] Chapman IT 2010 Phys Rev Lett 105255002

[34] Bussac MN and Pellat R 1987 Phys. Rev. Lett. 592650

[35] Gimblett CG and Hastie RJ 1994 Plasma Phys. Control. Fusion 361439

[36] Chu TK 1988 Nucl. Fusion 281109

[37] Hirshman SP, Van Rij WI and Merkel P 1986 Comput. Phys. Commun. 43143

[38] Anderson DV et al 1990 Int. J. Supercomp. Appl. 434

[39] Cooper WA et al 2010 accepted Plasma Phys. Control. Fusion

[40] Boozer AH, 2001 Phys. Rev. Lett. 865059

[41] Reimerdes H et al 2004 Phys. Rev. Lett. 93135002

[42] Mikhailovskii AB, Huysmans GTA, Sharapov SE and Kerner WO 1997 Plasma Phys. Rep. 23844

[43] Gryaznevich MP et al 2008 Plasma Phys. Control. Fusion 50124030

[44] Lanctot M et al 2010 Phys Plasmas 17030701

[45] Liu YQ, Chapman IT, Saarelma S, Gryaznevich MP, Hender TC and Howell DF 2009 Plasma Phys. Control. Fusion $\mathbf{5 1} 115005$

[46] Strait EJ et al 1995 Phys. Rev. Lett. 742483

[47] Hender TC et al. in Fusion Energy 2006 (Proc. 21st Int. Conf. Chengdu, 2006) (Vienna: IAEA) CD-ROM file EX/P8-18 and http://wwwnaweb.iaea.org/napc/physics/FEC/FEC2006/html/index.htm

[48] Sabbagh SA et al 2006 Nucl. Fusion 46635

[49] Garofalo AM et al 2002 Phys. Plasmas 91997

[50] La Haye RJ et al 2004 Nucl. Fusion 441197

[51] Sontag AC et al 2005 Phys. Plasmas 12056112

[52] Reimerdes $\mathrm{H}$ et al 2006 Phys. Plasmas 13056107

[53] Fitzpatrick R and Hender TC 1991 Phys. Fluids B3 644

[54] Reimerdes H et al 2007 Phys. Rev. Lett. 98055001

[55] Takechi M et al 2007 Phys. Rev. Lett. 98055002

[56] Strait EJ et al 2007 Phys. Plasmas 14056101

[57] Sabbagh SA et al. in Fusion Energy 2008 (Proc. 22nd Int. Conf. Geneva, 2008) (Vienna: IAEA) CD-ROM file EX/5-1 and http://wwwnaweb.iaea.org/napc/physics/FEC/FEC2008/html/index.htm

[58] Rice J et al 2007 Nucl. Fusion 471618

[59] Bondeson A and Ward DJ 1994 Phys. Rev. Lett. 722709

[60] Chu MS et al 1995 Phys. Plasmas 22236

[61] Hu B, Betti R and Manickam J 2005 Phys. Plasmas 12057301

[62] Hu B and Betti R 2004 Phys. Rev. Lett. 93105002

[63] Chapman IT et al 2009 Plasma Phys. Control. Fusion 51055015

[64] Liu YQ et al, 2009 Phys. Plasmas 16056113

[65] Liu YQ et al, 2008 Phys. Plasmas 15092505

[66] Berkery J et al, 2010 Phys. Rev. Lett. 104035003

[67] Liu YQ, 2010 Nucl Fusion 50095008

[68] Berkery J et al, 2010 Phys. Plasmas 17082504

[69] Breizman BN, Candy J, Porcelli F and Berk H 1998 Phys. Plasmas 52326 
[70] Liu YQ et al 2008 Phys. Plasmas 15112503

[71] Pinches SD et al 1998 Comput. Phys. Commun. 111133 (Release Version 8.09)

[72] Graves JP et al 2010 Phys. Plasmas 17056118

[73] Huysmans GTA and Czarny O, 2007 Nucl Fusion 47659

[74] Huysmans GTA, 2005 Plasma Phys. Control. Fusion 472107

[75] Sato M and Nakajima N, 2006 Phys Plasmas 13102507

[76] Sato M and Nakajima N, 2006 J. Plasma Physics 721223

[77] Matsunaga G et al, 2010 Nucl Fusion 50084003

[78] Okabayashi M et al. 2008 22nd IAEA FEC, Geneva $\mathbf{E X / 5 - 2}$

[79] Matsunaga G et al. 2008 22nd IAEA FEC, Geneva EX/P9-5

[80] Stork D et al. 2010 23rd IAEA FEC, Daejeon ICC/P5-06

[81] Voss G et al 2008 Fusion Eng. Des. 83 1648-53

[82] Hole MJ, Wilson HR, Abeysuriya R and Larson JW 2010 Plasma Phys. Control. Fusion 52125005

[83] Chapman IT et al 2007 Plasma Phys Control Fusion 49 B35

[84] Chapman IT 2011 Plasma Phys Control Fusion 53013001

[85] Chapman IT et al 2009 Phys. Plasmas 16072506 